\title{
"Không tìm mà thấy" trong hoạt động khởi nghiệp
}

\author{
Nguyễn Tô Hồng Kông \\ Trường Đại học Phú Xuân \\ Huế, Việt Nam
}

\section{Tóm tắt:}

Bài luận này nhằm phân tích và thảo luận liệu phương pháp "serendipity" có thể thực sự trở thành một lợi thế chiến lược trong hoạt động khởi nghiệp, dựa trên quan điểm của Napier \& Vuong (2013) hay không, và nếu có (hoặc không) thì có thể định hướng suy nghĩ về loại giá trị "không tìm mà thấy" đó như thế nào?

Keywords: 'Serendipity', lợi thế chiến lược, hoạt động khởi nghiệp

\section{Về bối cảnh mới của nghiên cứu khởi nghiệp}

Hoạt động khởi nghiệp là một bộ phận quan trọng trong quá trình phát triển kinh tế của Việt Nam, góp phần đem lại sức sống cho khu vực kinh tế phi nhà nước, tạo ra việc làm và sản lượng hàng hóa-dịch vụ đáp ứng nhu cầu xã hội (Vuong, Napier, Do, \& Vuong, 2016), ngay cả trong những thời kỳ khó khăn nhất, trước và sau Đổi Mới (Hoang \& Dung, 2009; Hoang et al., 2011).

Tuy nhiên, chất lượng của hoạt động khởi nghiệp trong nền kinh tế luôn là một câu hỏi quan trọng, vì ngày càng xuất hiện nhiều ràng buộc về văn hóa (Hoang \& Dung, 2009; Vuong, 2016a; Vuong et al., 2018) và hiệu suất kinh doanh của doanh nghiệp nhỏ, do hiện tượng khuynh hướng lợi suất giảm dần (Vuong, 2016b; Vuong \& Napier, 2014; Vuong, Napier, Tran, \& Nguyen, 2013), và quá trình tích tụ vốn chứa đựng nhiều rủi ro (Vuong, 2014).

Tổn thất kinh tế do việc tìm kiếm cơ hội thị trường ngày càng tăng lên, một phần do mặt bằng giá tăng lên qua thời gian (Vuong, 2014; Vuong, 2019), phần khác do hiệu quả của quá trình đó bị hạn chế bởi các xung đột lợi ích cũng như thiếu khả năng tối ưu hóa việc sử dụng nguồn nhân tài vật lực cho một số mục tiêu trọng điểm (Vuong \& Napier, 2014).

Tình hình đó dẫn đến câu hỏi liệu có cách nào cải thiện, hoặc tạo thêm lựa chọn, cho chiến lược tiếp cận cơ hội thị trường cho các doanh nghiệp khởi nghiệp, vốn ở quy mô nhỏ và siêu nhỏ?

Phân tích tiếp theo không nhằm trả lời câu hỏi lớn trên, mà trình bày một hướng tiếp cận khả dĩ, có tên gọi khá nghệ sỹ là "không tìm mà thấy", được đề xuất 
cách đây hơn 5 năm bởi Napier \& Vuong (2013) dưới thuật ngữ "serendipity", gắn với ý niệm lợi thế chiến lược trong đời sống kinh tế, trong cuốn Strategic Management in the 21st Centure (Praeger/ABC-Clio).

\section{Một số quan sát}

Có thể thấy giá trị của tiếp cận "không tìm mà thấy" (serendipity), đang ngày càng được quan tâm nhiều hơn trong các nghiên cứu và cả chính sách trong khoảng thời gian 5 năm trở lại đây.

Nhiều nhà nghiên cứu nhìn thấy giá trị chiến lược của tiếp cận này trong việc đào tạo ra một năng lực khó nắm bắt: sáng tạo (Makri, Ravem, \& McKay, 2017; Papaleontiou-Louca, Varnava-Marouchou, Mihai, \& Konis, 2014; Koivisto, 2017).

Nhưng rõ rệt nhất vẫn là với hiệu quả của việc nắm bắt và sử dụng thông tin trong thời đại bùng nổ công nghệ (Bell \& Cooper, 2015; DeRosa, McElwee, \& Smith, 2019; McCay-Peet \& Toms, 2017; Solomon \& Bronstein, 2016).

Với giá trị được nhận ra giàu tính chiến lược, Sethna (2017) đã đưa ra đánh giá tổng quan về giá trị của "serendipity" trong nhận thức quan trọng mới về tiếp cận cơ hội thị trường, trong đó tiếp cận "lợi thế chiến lược" được liệt kê trong danh mục các ý niệm nghiên cứu chủ chốt.

Với mức độ gia tăng thông tin và tốc độ dòng chảy trong thế giới hiện đại, loài người nói chung, và giới công thương, khởi nghiệp nói riêng, đang tiệm cận một mức độ hỗn loạn nhất định về thông tin, và số lượng cơ hội thực sự có thể được khai thác nhờ lợi thế chiến lược của "serendipity" trở nên nhỏ bé về tỷ trọng, và thời gian tồn tại trong vùng quan sát được trở nên ngắn ngủi. Mặt khác, khi mức độ hỗn loạn tiếp tục tăng lên, việc "tìm để thấy" trở nên vô cùng khó khăn, và xác suất để tìm nhầm cũng tăng mạnh. Như vậy, hoạt động khởi nghiệp đứng trước bài toán nan giải vì hiệu quả của "không tìm mà thấy" giảm đi nhanh chóng, nhưng "tìm để thấy" trở nên rất bất trắc khiến cho rủi ro tăng mạnh.

Nghịch lý này có lời giải cho giới khởi nghiệp không?

Chúng ta có thể quan sát một hiện tượng khác, có ý nghĩa tương tự về mức độ rủi ro, thách thức và sự bất ổn định trật tự ở thời kỳ đầu Đổi Mới. Đó là thời kỳ mà được cho là phản ánh các giá trị phổ biến của các "chính trị gia khởi nghiệp" (Vuong, 2014). Một trong những yếu tố rất quan trọng của hệ giá trị này chính là việc nhìn thấy giá trị của một số mô hình kinh tế tiềm tàng có tác động tích cực sâu rộng và đủ bền để trở thành chính sách trong khoảng thời gian kế tiếp.

Giá trị đó dần dần đã trở thành lợi thế chiến lược, do các vấn đề mà giai đoạn chuyển đổi kinh tế thị trường tiếp theo phải đối mặt đều không có tiền lệ, và các giải pháp đều phải dựa vào dòng thông tin mới xuất hiện, và tận dụng các giải pháp được nhận thấy công năng trên đường đi. Nói cách khác, không có một hệ thống giải pháp có sẵn, dựa trên những lời giải của mô hình cơ bản là cố định.

Những đặc trưng nói trên chỉ ra một thực tế: Hệ thống khởi nghiệp hoàn toàn có thể tạo ra chiến lược thích nghi dựa trên nguyên lý lợi thế chiến lược của "serendipity".

\section{Thay cho kết luận}


Tuy nhiên, để có thể khai thác lợi thế chiến lược vừa bàn ở trên, thời đại thông tin đòi hỏi một tiếp cận hệ thống hơn với phương pháp. Để có thể nhìn ra tính khả thi của tiếp cận này cho hoạt động khởi nghiệp, sẽ cần có một khảo sát tổng quan lý thuyết, dựa trên các bằng chứng thống kê của giai đoạn 30 năm vừa qua tại Việt Nam. Công việc này giúp nhìn thấy rõ hơn ảnh hưởng thực tế, và những thông tin còn thiếu cần bổ sung cho đánh giá hoàn chỉnh hơn về tác dụng và tính khả thi của "không tìm mà thấy".

Tiếp theo, nhưng các nghiên cứu đã đóng góp nhận thức, mọi hiện tượng và hiệu năng của phương pháp tiếp cận (hay lời giải tình huống) đều bị ràng buộc và hạn chế bởi các điều kiện kinh tế-xã hội. Những điều kiện đó thay đổi qua từng giai đoạn, cho dù cơ bản vẫn giữ đặc trưng, nên sẽ tác động đáng kể tới hiệu lực của một tiếp cận chiến lược đối với giá trị khai thác cơ hội khởi nghiệp như "serendipity". Không nhận dạng rõ được các điều kiện đó tức là cũng không trả lời được câu hỏi về tính phù hợp cũng như không dự báo được mức độ tác động tới cải thiện hiệu quả kinh tế.

\section{References:}

Bell, V., \& Cooper, S. (2015). Serendipity in international entrepreneurial opportunities. Proceedings ACERE 2015, Australian Centre for Entrepreneurship Research Exchange Conference 2015, 3-6 February, Adelaide, South Australia.

DeRosa, M., McElwee, G., \& Smith, R. (2019). Farm diversification strategies in response to rural policy: a case from rural Italy. Land Use Policy, 81, 291-301.

Hoang, V. Q., \& Dung, T. T. (2009). The cultural dimensions of the Vietnamese private entrepreneurship. IUP J. Entrepreneurship Development, 6(3\&4), 54-78.

Hoang, V. Q., Van Nhue, D., Van Houtte, D., \& Dung, T. T. (2011). The Entrepreneurial facets as precursor to Vietnam's economic renovation in 1986. IUP Journal of Entrepreneurship Development, 8(4), 6-47.

Koivisto, E. (2017). Leveraging serendipity in trade shows. ANZMAC 2017 Proceedings, 4-6 December, Melbourne, Australia.

Makri, S., Ravem, M., \& McKay, D. (2017). After serendipity strikes: Creating value from encountered information. Proceedings of the Association for Information Science and Technology, 54(1), 279-288.

McCay-Peet, L., \& Toms, E. G. (2017). Researching serendipity in digital information environments. Synthesis Lectures on Information Concepts, Retrieval, and Services, 9(6), i-91.

Napier, N. K., \& Vuong, Q. H. (2013). Serendipity as a strategic advantage?. In Wilkinson (Ed.) Strategic Management in the 21st Century (Vol. 1: The Operational Environment, pp. 175-199). Westport, CT: Praeger/ABC-Clio.

Østergaard, A. (2014). Entrepreneurial Leadership Talent-an empirical research for keyfactors. In DRUID Society Conference, CBS, Copenhagen, June 16-18. 
Papaleontiou-Louca, E., Varnava-Marouchou, D., Mihai, S., \& Konis, E. (2014). Teaching for creativity in universities. Journal of Education and Human Development, 3(4), 131-154.

Sethna, Z. (2017). Editorial: New perspectives on digital marketing, social entrepreneurship and serendipity in entrepreneurial marketing. Journal of Research in Marketing and Entrepreneurship, 19(2), 201-206, DOI: 10.1108/JRME-11-2017-0048.

Solomon, Y., \& Bronstein, J. (2016). Serendipity in legal information seeking behavior: Chance encounters of family-law advocates with court rulings. Aslib Journal of Information Management, 68(1), 112-134.

Vuong, Q. H. (2014). Vietnam's political economy: a discussion on the 1986-2016 period. WP-CEB N $14-010$, Université Libre de Bruxelles.

Vuong, Q. H. (2016a). Impacts of geographical locations and sociocultural traits on the Vietnamese entrepreneurship. SpringerPlus, 5(1), art. 1189, DOI: 10.1186/s40064-016-2850-9.

Vuong, Q. H. (2016b). Determinants of firm performance in a less innovative transition system: exploring Vietnamese longitudinal data. International Journal of Transitions and Innovation Systems, 5(1), 20-45.

Vuong, Q. H. (2019). The financial economy of Viet Nam in an age of reform, 19862016. In U. Volz, P. Morgan and N. Yoshino (Eds.) Routledge Handbook of Banking and Finance in Asia (pp. 201-222). New York, NY: Routledge.

Vuong, Q. H., La, V. P., Vuong, T. T., Nguyen, V. H., Ho, M. T., Nguyen, T. H. K., Bui, Q. K., \& Ho, M. T. (2018). Cultural additivity: Behavioural insights from the interaction of Confucianism, Buddhism, and Taoism in folktales. Palgrave Communications, 4, DOI: 10.1057/s41599-018-0189-2.

Vuong, Q. H., \& Napier, N. K. (2014). Resource curse or destructive creation in transition: evidence from Vietnam's corporate sector. Management Research Review, 37(7), 642-657.

Vuong, Q. H., Napier, N. K., Do, T. H., \& Vuong, T. T. (2016). Creativity and entrepreneurial efforts in an emerging economy. Business Creativity and the Creative Economy, 2(1), 39-50. DOI: 10.18536/bcce.2016.10.2.1.04.

Vuong, Q. H., Napier, N. K., Tran, T. D., \& Nguyen, T. H. K. (2013). A categorical data analysis on financial failures in Vietnam, 2007-2013. International Journal of Business and Management, 8(8), 87-94.

Vuong, Q. H., Vu, Q. H., \& Vuong, T. T. (2016). Relationship between past experience, social network participation and creative capacity: Vietnamese entrepreneurship in transition. International Journal Transitions and Innovation Systems, 5(3/4), 299-313. 